Research article

2

\title{
Whole-cell biosensor of cellobiose and application to wood decay detection
}

4 Maxime Toussaint ${ }^{1,2}$

5 Cyril Bontemps ${ }^{1,2^{*}}$

6 Arnaud Besserer ${ }^{3}$

7 Hotel Laurence ${ }^{1,2}$

8 Philippe Gérardin ${ }^{3}$

9 Pierre Leblond ${ }^{1,2^{*}}$

${ }^{1}$ Laboratoire Dynamique des Génomes et Adaptation Microbienne, UMR 1128, Université de Lorraine, Vandœuvre-lès-Nancy, F-54506, France

${ }^{2}$ Laboratoire Dynamique des Génomes et Adaptation Microbienne, UMR 1128, INRA, Vandœuvre-lès-Nancy, F-54506, France

${ }^{3}$ Laboratoire d'Etudes et de Recherches sur le Matériau Bois, EA4370, Université de Lorraine USC INRA, Faculté des Sciences et Technologies, 54506, Vandœuvre-lès-Nancy, F-54506, France

Correspondence $\left(^{*}\right)$ : Dr. Cyril Bontemps, Laboratoire Dynamique des Génomes et Adaptation Microbienne, UMR 1128, INRA-Université de Lorraine, Faculté des Sciences et

Technologies-Campus Aiguillettes, 54506 Vandœuvre-lès-Nancy, France

Pr. Pierre Leblond, Laboratoire Dynamique des Génomes et Adaptation Microbienne, UMR 1128, INRA-Université de Lorraine, Faculté des Sciences et Technologies-Campus Aiguillettes, 54506 Vandœuvre-lès-Nancy, France

E-mail : cyril.bontemps@univ-lorraine.fr pierre.leblond@univ-lorraine.fr

Keywords: Biosensor, CebR, cellobiose, Streptomyces, synthetic biology, wood decay 


\section{Abstract}

32 Fungal biodegradation of wood is one of the main threats regarding its use as a material. So far, the detection of this decaying process is empirically assessed by loss of mass, when the fungal attack is advanced and woody structure already damaged. Being able to detect fungal attack on wood in earlier steps is thus of special interest for the wood economy. In this aim, we designed here a new diagnostic tool for wood degradation detection based on the bacterial whole-cell biosensor technology. It was designed in diverting the soil bacteria Streptomyces CebR sensor system devoted to cellobiose detection, a cellulolytic degradation by-product emitted by lignolytic fungi since the onset of wood decaying process. The conserved regulation scheme of the CebR system among Streptomyces allowed constructing a molecular tool easily transferable in different strains or species and enabling the screen for optimal host strains for cellobiose detection. The selected biosensor was able to detect specifically cellobiose at concentrations similar to those measured in decaying wood and in a spruce leachate attacked by a lignolytic fungus, indicating a high potential of applicability to detect ongoing wood decay process. 
Thanks to its intrinsic mechanical properties, wood is used since the Neolithic era as a material for construction, furnishing, heating or wood-derived products such as paper. Nowadays, there is also a regain of interest for this material as it is renewable and a source of biomass for conversion into bio-ethanol [1]. As a material, wood is highly resistant to the environmental conditions such as rain, sun or other abiotic stresses and the main threat regarding its use comes mostly from the attack by lignolytic organisms. This wood biodegradation represents a major economic problem. For instance, in the US only, it costs more than 5 billion USD each year to homeowners and almost $10 \%$ of the annual product of forest is used to replace the degraded products [2]. Being able to detect the early wood biodegradation is thus crucial. During storage, it would avoid to use contaminated wood for construction. When the wood is already used as a material it would enable to apply early curative measures before any significant loss of mechanical properties. However, to our knowledge, there is no tool available to achieve this goal and wood degradation is so far only assessed by resistance testing and visual expertise only relevant and operating on wood decayed at an advanced level.

Wood bio-degradation in forest ecosystems is mainly the fact of diverse fungi [3] classified according to the type of decay they cause (e.g. white-rots, brown-rots, soft-rots, see for review [4]). If rot-fungi have distinct specificities in term of ecology or degradation strategies, they nevertheless all degrade and use as a carbon source the cellulose that represents $40 \%$ to $50 \%$ of the plant dry weight [5]. Two main degradation strategies exist during the wood degradation by fungi: enzymatic pathways involving cellulases or oxidative mechanisms using Fenton reaction and polysaccharide monooxygenases [6,7]. However, a canonical degradation pathway is common for all of them where the cellulose fiber is shortened into simpler forms: the cellobiose (two $\beta-1,4$ linked glucose units) or sometimes into cellodextrines (generally from three to six $\beta$-1,4-linked glucose units) $[7,8]$. These cellulose degradation by-products will be hydrolyzed later on into glucose by the action of $\beta$ glucosidases [8]. Thus, the initial release and presence of cellobiose and cellodextrines is a common denominator and can be considered as a signature of the attack of wood by rotfungi.

The Streptomyces are filamentous spore forming soil dwelling bacteria that are generally not able to degrade native wood, but are considered as essential to recycle biomass polymers in environment thanks to their large-enzymatic arsenal able to degrade wood by-products $[9,10]$. The detection of these compounds and the activation of the enzymatic pool to degrade them has been linked to the regulator CebR of the Lacl family in Streptomyces 
griseus [11], Streptomyces reticuli [12], Streptomyces sp. ActE [13] and Streptomyces scabies [14]. Since its characterization by Schlösser et al. [12], and Marushima et al. [11], it is known that the CebR transcriptional repressor prevents gene expression from binding a conserved 22 bp hairpin motif (cebR-box) found in the transcriptional region of its targets. In presence of inducer molecules such as cellobiose or in some cases cellodextrines or cellulose, the CebR repression is alleviated and enables the expression of the controlled genes. So far, the complete CebR regulome is not well-known. However, a transcriptomic analysis of the Streptomyces sp. ActE (Sirex) (a symbiotic strain that helps the pine-boring woodwasp Sirex noctilio to deconstruct wood biomass) has shown that, in presence of wood derived compounds, the most up-regulated genes were under the control of the CebRsystem. These genes were mostly involved in the uptake (ABC transporter system) or in the production of cellulolytic and hemicellulolytic enzymes ( $\beta$-glucosidases, cellulases, cellobiohydrolases, mannosidases) [13]. Moreover, CebR could induce other functions like the pathogenic factors of $S$. scabies in presence of cellobiose [15].

Since cellobiose is a key product of cellulose hydrolysis and indirectly indicates fungal wood degradation, we designed and developed a cebR-box based biosensor expressed by Streptomyces in order to detect the presence of cellobiose, demonstrated its sensibility and specificity and showed its applicability in a case of wood degradation detection.

\section{Material and Methods}

\section{1) Plasmids, strains and media}

Plasmids and strains used in this work are presented in Table 1. Escherichia coli strains were grown in LB medium [16] at $37^{\circ} \mathrm{C}$ and Streptomyces at $30^{\circ} \mathrm{C}$ either on solid SFM medium [20 g mannitol, $20 \mathrm{~g}$ soy flour and $20 \mathrm{~g}$ bacto-agar per liter] or in liquid modified HT medium $\left(\mathrm{HT}^{\star}\right)$ [ $1 \mathrm{~g}$ yeast extract, $1 \mathrm{~g}$ beef extract, $5 \mathrm{~g}$ mannitol, $2 \mathrm{~g}$ bacto-tryptone and $0.02 \mathrm{~g}$ $\mathrm{COCL}_{2}$ per liter, $\mathrm{pH}=7.3$ ]. For the biosensor tests, cellobiose (Alfa Aesar, Karlsruhe, Germany) and cellodextrines (Elicityl, Crolles, France) were added after autoclaving in liquid modified HT at the appropriate concentration. Avicel Ph-101 (Sigma, St. Louis, USA), a crystalline form of cellulose, was added in liquid medium before autoclaving. Media were supplemented with apramycin $\left(50 \mu \mathrm{g} \cdot \mathrm{ml}^{-1}\right)$ for the selection of strains transformed with the pIB139 derived plasmids or with ampicilline $\left(100 \mu \mathrm{g} \cdot \mathrm{ml}^{-1}\right)$ for the selection of the $E$. coli transformed with pGemT-easy (Promega, Madison, USA). 
Wood degradation was performed according the European standard EN 113, 1996 [17] with some modifications. Spruce (Picea abies L.) wood specimens $(40 \times 15 \times 5 \mathrm{~mm})$ were sterilized at $103^{\circ} \mathrm{C}$ for 2 days. Then wood specimens were placed under sterile conditions in petri dishes containing one-week old mycelium of the lignolytic Poria placenta fungus. Wood specimens were incubated at $22^{\circ} \mathrm{C}$ and $70 \%$ relative humidity for 16 weeks. For leachate preparation, $P$. placenta mycelium was carefully removed from the wood by hand scratching. Eight wood specimens were impregnated under vacuum with $100 \mathrm{ml}$ of PBS buffer $\mathrm{pH} 7.4$ for $15 \mathrm{~min}$. For cellobiose quantification, $20 \mu \mathrm{l}$ of leachate were run on HPLC equipped with a refractometer. Sugars were separated on a Shodex sugar KS-803 column (Waters SAS, Guyancourt, France) at $80^{\circ} \mathrm{C}$ with a flow of $0.8 \mathrm{~mL} \cdot \mathrm{min}^{-1}$ using HPLC grade water as solvent. Cellobiose and glucose were detected after $12.4 \mathrm{~min}$ and $13.2 \mathrm{~min}$ of elution, respectively. The cellobiose concentration present in the leachate was estimated by peak area integration and comparison with a cellobiose standard calibration curve. The cellobiose calibration curve ranged from $2.92 \mathrm{mM}$ to $29.3 \mu \mathrm{M}$. Specificity and detection of free glucose resulting from wood cell wall degradation was detected by comparison with a calibration curve build from glucose concentrations ranging from $5.56 \mathrm{mM}$ to $55.6 \mu \mathrm{M}$.

Polymerase chain reactions were performed with the Dream Taq polymerase (ThermoFisher Scientific, Waltham, USA) for fragments under $1.5 \mathrm{~kb}$ in size or Taq polymerase Takara (Takara Bio Inc., Kusatsu, Japan) for larger fragments. PCR primers are listed in Table S1. Ligation reactions were carried out with T4 DNA ligase (ThermoFisher Scientific, Waltham, USA). DNA was digested and purified from gel matrix respectively with restriction enzymes and the GeneJet Gel Extraction Kit purchased from ThermoFisher Scientific (Waltham, USA). The kits were used according to supplier's recommendations. Alkaline lysis plasmid extractions, plasmid electroporations and DNA extractions were performed as described in [18].

\section{4) Functional characterization of biosensors}

145 Functional screenings of the biosensors efficiency were performed by streaking of $2 \mu \mathrm{L}$ of 146 spore suspension (ca $10^{8}-10^{9}$ spores $/ \mathrm{mL}$ ) of each biosensor on $\mathrm{HT}^{*}$ plates supplemented with $5 \mathrm{~g} \cdot \mathrm{L}^{-1}$ of cellobiose and incubation at $30^{\circ} \mathrm{C}$ for 2 days. Controls without cellobiose or 
with crystalline cellulose (Avicel) were performed in parallel. After growth, plates were sprayed with a $0.5 \mathrm{M}$ catechol solution and incubated for 10 to $20 \mathrm{~min}$ in the dark. The visualization of a yellow color in the mycelium and in its surrounding medium attested the detection of cellobiose by the biosensor after confrontation with the control plates i.e. without cellobiose and supplemented with crystalline cellulose. A positive result in both latter cases would constitute false positives.

\section{5) Biosensor microplate tests}

Spore suspensions of the Streptomyces S4N27 biosensor strain were realized on SFM complemented with apramycin [16] and stored in a $20 \%(\mathrm{v} / \mathrm{v})$ glycerol solution at $-20^{\circ} \mathrm{C}$. To perform biosensor qualitative and quantitative tests in presence of cellobiose, cellodextrines or wood leachates, $5.10^{5}$ spores were inoculated into $3 \mathrm{ml}$ of modified HT medium cultures complemented with the sugars at the selected concentrations or with $1 \mathrm{ml}$ of wood leachate. Cultures were incubated at $30^{\circ} \mathrm{C}$ for $16-18$ hours under agitation (200 rpm). Cells were harvested by centrifugation at $4,500 \mathrm{rpm}$ for $5 \mathrm{~min}$, washed with $1 \mathrm{ml}$ of $20 \mathrm{mM}$ phosphate buffer $\mathrm{pH} 7.2$ and suspended in $1 \mathrm{ml}$ of sample buffer (100 mM phosphate buffer pH 7.5, 20 mM NaEDTA pH 8, 0.1\% Triton X100, 10\% acetone [v/v]). Cells were then lysed on ice by sonication (three cycles of $20 \mathrm{~s}$ in Bioruptor Standard device, Diagenode, New Orleans, USA). The cell lysate was harvested by centrifugation for $6 \mathrm{~min}$ at $7,000 \mathrm{rpm}$ at $4^{\circ} \mathrm{C}$ and $20 \mu \mathrm{l}$ were mixed with $300 \mu \mathrm{l}$ of assay buffer (100 mM phosphate buffer $\mathrm{pH} 7.5,0.2 \mathrm{mM}$ cathecol) previously warmed at $30^{\circ} \mathrm{C}$. Assay buffer was prepared just before use by adding the catechol from a $20 \mathrm{mM}$ stock solution (in water) to the pre-warmed phosphate buffer [19] .

The yellow catechol degradation signal resulting from XylE activity was quantified by spectrometry at OD $595 \mathrm{~nm}$ (Bioteck, Winooski, USA). The catechol dioxygenase activity was determined and normalized at $375 \mathrm{~nm}$ per min per milligram of protein and converted to milliunits per milligram $[16,19]$. The protein concentration was estimated by the method of Bradford [20] by using bovine serum albumin as a standard. For each condition, experiments were realized with at least three repeats. The result of each experiment was expressed as the expression of XylE in the assay relative to that observed in the controls (i.e., in the absence of cellobiose) systematically run in parallel. The significance of the relative signal of the assay versus the controls was statistically assessed with a $t$-student test. 


\section{Results}

\subsection{In silico analysis to assess the potential of Streptomyces as biosensor} hosts

CebR prevents gene transcription in binding a specific sequence called the cebR-box and this repression is alleviated by the recognition of the inducer molecules, i.e. the cellobiose (Figure 1A). The CebR sensor system has been previously found and reported as a highly conserved regulatory system in some Streptomyces species [11-13], notably in term of sequence identity of the cebR-box. The concept of the biosensor was to build a generic biosensor cassette that could be regulated in different genetic backgrounds, i.e. in Streptomyces strains potentially exhibiting different specificities or sensitivities towards the target molecules. In order to assess the range of utilization of this construction within the Streptomyces genus we investigated the distribution of the CebR system within the 42 available fully sequenced genomes. BlastP searches (NCBI) were done using as query the CebR sequence of the fully characterized Streptomyces reticuli system CebR (CUW28692) [12] with a cut-off of $50 \%$ of identity in amino acid. Conserved regulatory motif TGGGAGCGCTCCCA the so-called "cebR-box" was also searched. All genomes but five (Streptomyces albulus NK660, Streptomyces albulus ZPM, Streptomyces albus DSM41398 and Streptomyces sp. 769, Streptomyces lydicus A02) possessed of at least one CebR homologue with amino acid identity ranging from $63 \%$ to $96 \%$ and the cebR-box motif as well as conserved regulatory cebR-boxes. This analysis showed that the CebR-system is widespread in this genus and conserved enough to recognize similar cebR-boxes. Consequently, most of the Streptomyces strains (including presumably environmental ones) are likely to be able to regulate a construction based on a conserved cebR-box through their endogenous CebR protein and could be turned into biosensors.

\subsection{Construction of the biosensor}

208

209

210

The biosensor design is depicted in Figure 1B. Briefly, the concept consists to put a reporter gene under the transcriptional control of CebR. The xylE gene from Pseudomonas putida which was successfully used in Streptomyces was chosen $[16,19]$. It codes for a catechol dioxygenase that converts the colorless substrate catechol to an intensely yellow hydroxymuconic semialdehyde. The genomic DNA of Streptomyces coelicolor KC900 which includes a $x y / E$ gene was used as a PCR template with the primer couple consisting of the forward Biosens_F and the reverse Biosens_R primers (Table S1, Figure 1B.I). The 
Biosens_F primer sequence includes a cebR-box and a ribosome binding site (RBS). The amplification thus places the cebR-box and a RBS site upstream of the coding region of $x y / E$ (Figure 1B.I). The 955 bp PCR product was subcloned in the pGEM-T Easy vector, introduced in $E$. coli $\mathrm{DH} 5 \alpha$ and its sequence checked by sequencing. After releasing from pGEM-T Easy by a double restriction digestion BamHI-EcoRI present in the Biosens_F and Biosens_R primer sequences respectively. This DNA fragment was further cloned directionally into the multicloning site of the pIB139 vector also digested with the same couple of endonucleases (Figure 1B.II). This cloning step resulted in the positioning of the $x y I E$ gene downstream the strong constitutive promoter PermE ${ }^{\star}[21,22]$. This construct could then be mobilized into Streptomyces cells by intergeneric conjugation using the intermediary of $E$. coli S.17 host strain. The vector plB139 was chosen for its site-specific integration system targeting the bacteriophage $\Phi C 31$ attachment site [16]. The integration into the chromosome secures the stability of the construct. A single copy is expected to be inserted.

Nine Streptomyces species were chosen as receptors and the insertion of the construct was selected by the presence of apramycin in the culture medium. The absence of endogenous catechol dioxygenase activity was checked on plate in presence of catechol which is the XyIE substrate (data not shown). Three of them were reference strains (Streptomyces ambofaciens ATCC 23877, Streptomyces coelicolor M145 and Streptomyces lividans TK23). The seven others were environmental strains (Streptomyces sp. S9N29, S2N2, S6N6, S9N14, S4N22, S1N3 and S4N27). These strains were chosen for their cellulolytic properties and their representativeness of the taxonomic diversity of a forest soil [9]. The presence of CebR was expected and highly likely considering the wide distribution of the CebR system in sequenced genomes (see above). After conjugation with E. coli S17.1 harboring the biosensor construct, Streptomyces transconjugants were selected on SFM plates with apramycin and nalidixic acid which counter selects E. coli. For each Streptomyces strain, eight transconjugants were selected. The integration into the $\Phi$ C31 attachment site was checked by PCR for S. ambofaciens ATCC 23877. For environmental strains, the insertion of the construct was checked by PCR amplification of the xylE gene (data not shown).

\subsection{Screening for optimal host strain for the biosensor}

In order to confirm the efficiency of the constructed strains as biosensors, they were functionally tested for their ability to emit a yellow color on plates complemented with cellobiose $\left(5 \mathrm{~g}^{-\mathrm{L}^{-1}}\right)$ as an inducer molecule. In comparison with plates without cellobiose, all transformed clones for 5 strains (S. ambofaciens ATCC 23877, S. coelicolor M145, S.lividans TK24, Streptomyces S4N27 and Streptomyces S6N6) exhibited a bright yellow coloration of mycelium and in the surrounding solid medium, indicating that the cellobiose was able to 
unlock the CebR repression and to activate the expression of $x y / E$. In order to choose the optimal host strain among them, several screenings were performed (Table S2) and the strain S4N27 was selected for the following reasons. First, it visually exhibited the highest yellow color on plate (Figure 2) suggesting an optimal production of the XylE protein. Secondly, no detection signal was obtained in presence of crystalline cellulose (Avicel), indicating that the presence of none-degraded cellulose could not lead to false positive. Further specificity and sensitivity experiments were carried out on mycelium grown in liquid medium in order to ensure quantitative and reproducible assays. For that purpose, mycelium samples were sonicated to release the intracellular XylE content. Consequently, another selection criterion was the ability to lyse the mycelium. One of our candidate environmental Streptomyces strain, S4N27, was typified by a planktonic growth mode while other species grew as typical dense mycelium pellets difficult to lyse. For that reason, we finally selected strain S4N27 as the best host biosensor for further tests (Table S2).

\subsection{Sensitivity and specificity of cellobiose detection}

Tests were performed in liquid cultures to enable the quantification of the XylE activity of the biosensor S4N27 in presence of cellobiose. Its sensitivity was tested with cellobiose concentrations ranging from $1.5 \mu \mathrm{M}$ to $15 \mathrm{mM}$ and after $10 \mathrm{~min}$ of reaction. Controls without cellobiose were performed for each experiment and exhibited a background signal presumably corresponding to the basal $x y / E$ expression from the construction as no endogenous dioxygenase activity was observed in wild-type strains devoided to the construction. As the latter signal was equivalent in all controls, the xylE activity of the assays was expressed as a relative expression of this baseline and did not raise an issue regarding the interpretations of the results. A significant detection was observed starting from $15 \mu \mathrm{M}$ of cellobiose (equivalent to $5 \mathrm{mg} \cdot \mathrm{L}^{-1}$ concentration). This is in the same range than a leachate produced from spruce wood in degradation (see below) as measured by HPLC. However, no significant signal could be detected at $1.5 \mu \mathrm{M}$ (Figure 3). An increasing signal was observed in response to cellobiose concentration to reach a plateau from $80 \mu \mathrm{M}$. The stability of the colorimetric reaction was assessed every two minutes for $1 \mathrm{~h}$ at $80 \mu \mathrm{M}$ of cellobiose. Since 4 minutes and until the end of the experiment, all the measured intensity ratios were statically identical with a value close to 2.4 that showed there was no signal loss over time (data not shown). The chemical reaction was thus stable during this lap of time and biochemical instability of the reaction could not technically impair the liability of the measures.

At the same $80 \mu \mathrm{M}$ concentration of cellodextrines (triose, tetraose, pentaose and hexose), no signal could be observed (data not shown) showing that cellobiose was the specific signal sensed by the CebR system in the S4N27 strain. 
287 3.5 Application of the biosensor for wood degradation detection.

288 In order to test whether our biosensor was able to detect the presence of cellobiose in a 289 context of wood degradation, spruce sticks were infected by the lignolytic fungi Poria 290 placenta during a period of 7 weeks to allow an effective fungal attack. Wood leachates were 291 extracted and tested on the biosensor along with control leachates collected from uninfected 292 spruce sticks. The xylE expression for infected spruce was 1.8 relative to the control in the 293 same range than the ratio observed using pure cellobiose as inducer (Figure 4). The 294 cellobiose presence was checked and its concentration estimated in leachates by HPLC. 295 While no cellobiose was detected in the uninfected control, a concentration of $15 \mu \mathrm{M}$ was 296 measured in the infected wood. This result confirmed that the biosensor was able to detect a 297 low cellobiose concentration in a complex wood leachate and consequently enabled the 298 detection of the fungal decaying process. 
301

302

303

304

305

306

307

308

309

310

311

312

313

314

315

316

317

318

319

320

321

322

323

324

325

326

327

328

329

330

331

332

333

334

335

The aim of this study was to create a biosensor dedicated to cellobiose detection. We based its construction on a microbial cellobiose sensor: the Streptomyces CebR system. Since, its characterization [10,11], it is known that the CebR transcriptional repressor prevents gene expression from binding a conserved cebR-box. In presence of cellobiose it releases its action and enables the expression of the repressed genes such as genes coding cellulolytic enzymes [11,12] or pathogenic factors in S. scabies [15]. The presence of this system has been assessed in few Streptomyces so far [11-13,15], but thanks to an in silico prospection of the CebR-system, we showed that it was actually highly prevalent in this genus. We also showed that the CebR harboring Streptomyces all had highly conserved cebR-boxes, suggesting that their CebR regulators were prone to recognize similar ones.

We took advantage of this conserved genetic scheme to design a whole-cell biosensor. Most microbial sensors are generally based on a specific promoter cloned upstream a reporting gene $[23,24]$. Once integrated in the cell, the promoter initiates the transcription of the reporting gene when the cell comes in contact with the inducer signal. As CebR is a transcriptional repressor, we adapted this strategy in consequence and cloned a canonical $c e b R$-box in the promoter region of a constitutively expressed reporter gene in order to regulate its expression by CebR. The $c e b R$-box was cloned between the promoter and the transcription start of the gene as it is found in some Streptomyces [11]. Considering the widespread distribution of CebR among Streptomyces, we decided to integrate the construct in various Streptomyces and relied on the endogenous CebR for its regulation. As these CebRs were naturally present in the strains they had the advantage to be perfectly adapted to the recipient strain and thus to offer an optimal regulation of the construct. The construct was successfully transferred and integrated with the plB139 vector in different Streptomyces both model strains and environmental isolates, and its good regulation in presence of cellobiose was observed in one third of them. For the other strains, the absence of a visual signal during this screening could be due to various reasons such as a low xylE expression, a diffusion problem of the dioxygenase or non-recognition of cellobiose. All together, these results showed that our biosensor design was efficient and enabled to create a promiscuous construct that could be easily transferred and regulated in most Streptomyces to turn them into potential biosensors. More generally, this strategy that consists in diverting without $a$ priori a conserved sensor system in different strains to control a same conserved molecular construction could be probably applied to other systems and in other bacteria. A main advantage is that it allows for a same construction the screening of different strains with different sensitivity or sensibility towards the molecule of interest and might ease the construction of whole-cell biosensors. A further development to our approach could be to 
introduce the construction in a strain that does not harbor the CebR system (e.g. E. coli or a $c e b R$-free Streptomyces) along with a heterologously expressed cebR gene to control it. Previous biosensors, principally amperometric enzyme-based, were able to detect cellobiose [23-25]. They generally used cellobiose hydrolases that are catalytic enzymes able to degrade cellobiose. However, these enzymes have a large spectrum of molecule recognition and the biosensors built with them were used to detect other compounds such as glucose, lactose or catechol, but never specifically and directly the cellobiose [25]. Regarding the CebR system, some cross specificity for cellodextrines in addition to cellobiose has been identified in S. griseus [11]. Thus we did test the specificity of the Streptomyces S4N27 biosensor strain towards these compounds and got no positive detection, indicating a specificity of cellobiose recognition with our biosensor like in S. reticuli [12].

We designed the biosensor to detect wood degradation. Fungal attack is one of the main threats regarding the use of wood as a material. Generally, such attack is empirically discovered when the decaying process had already altered the wood mechanical properties. Even in the case of standardized tests aiming to assess the efficiency of protective treatments against fungal attack, professionals rely on 4 month experiments after infection where they measure the wood mass loss (EN 113) [17]. In order to assess whether our biosensor could bring a quicker answer in comparison to such tests, we performed a similar experiment as in the EN113 norm in infecting a spruce stick with Poria placenta, but for half of the normal time of the test. The application of the biosensor in this real context was successful and enabled to detect cellobiose in the wood attacked by the xylophagous fungus, but not in the uninfected control and by extension revealed the ongoing wood decaying process at this stage, dividing by two the time of the EN113 test. Such experiments are preliminary and despite further experiments are still needed to insure the full applicability of the biosensor in that context, this study led to a patent (PCT/EP2016/052417) regarding the exploitation of this biosensor. In future experiments, other types of wood-rots and kinds of wood should be tested but according to the fact that cellobiose remains a common denominator of fungal wood degradation process; it should be detected in most cases. Another aspect to be tested will be the kinetic of degradation to assess at which stage the biosensor would be applicable. Yet, as cellobiose is produced since the beginning of the decaying process, degradation of wood should be detected in the earlier steps.

In conclusion, we created here a whole-cell biosensor in diverting a widespread sensor of cellobiose in Streptomyces. According to the conservation of this system, we designed a construct optimized for being transferable and regulate by most Streptomyces. After selection of a biosensor strain, we have been able to detect cellobiose even in complex 
371 solutions such as a wood leachate. The application of this biosensor to detect wood 372 degradation appears thus very promising for the wood industry.

373 
[1] Wang, L., Littlewood, J., Murphy, R.J., Environmental sustainability of bioethanol production from wheat straw in the UK, Renew. Sustain. Energy Rev. 2013, 28, 715-725.

[2] Schultz, T.P., Nicholas, D.D., Introduction to Developing Wood Preservative Systems and Molds in Homes, Dev. Commer. Wood Preserv, American Chemical Society, 2008, pp. 2-8.

[3] Rajala T., Peltoniemi M., Pennanen T., Mäkipää R., Fungal community dynamics in relation to substrate quality of decaying Norway spruce (Picea abies [L.] Karst.) logs in boreal forests, FEMS Microbiol. Ecol. 2012, 81, 494-505.

[4] Schwarze, F.W.M.R., Engels, J., Mattheck, C., Fungal Strategies of Wood Decay in Trees, Springer Science \& Business Media, 2000.

[5] Howard, R.L., Abotsi, E., Van Rensburg, E.J., Howard, S., Lignocellulose biotechnology: issues of bioconversion and enzyme production, Afr. J. Biotechnol. 2004, 2,602-619.

[6] Phillips, C.M., Beeson, W.T., Cate, J.H., Marletta, M.A., Cellobiose dehydrogenase and a copper-dependent polysaccharide monooxygenase potentiate cellulose degradation by Neurospora crassa, ACS Chem. Biol. 2011, 6, 1399-1406.

[7] Lynd, L.R., Weimer, P.J., van Zyl, W.H., Pretorius, I.S., Microbial Cellulose Utilization: Fundamentals and Biotechnology, Microbiol. Mol. Biol. Rev. 2002, 66, 506-577.

[8] Langston, J.A., Shaghasi, T., Abbate, E., Xu, F., et al., Oxidoreductive cellulose depolymerization by the enzymes cellobiose dehydrogenase and glycoside hydrolase 61, Appl. Environ. Microbiol. 2011, 77, 7007-7015.

[9] Bontemps, C., Toussaint, M., Revol, P.-V., Hotel, L., et al., Taxonomic and functional diversity of Streptomyces in a forest soil, FEMS Microbiol. Lett. 2013, 342, 157-167.

[10] Bruce, T., Martinez, I.B., Maia Neto, O., Vicente, A.C.P., et al., Bacterial community diversity in the Brazilian Atlantic forest soils, Microb. Ecol. 2010, 60, 840-849.

[11] Marushima, K., Ohnishi, Y., Horinouchi, S., CebR as a Master Regulator for Cellulose/Cellooligosaccharide Catabolism Affects Morphological Development in Streptomyces griseus, J. Bacteriol. 2009, 191, 5930-5940.

[12] Schlösser, A., Aldekamp, Schrempf, T., H., Binding characteristics of CebR, the regulator of the ceb operon required for cellobiose/cellotriose uptake in Streptomyces reticuli, FEMS Microbiol. Lett. 2000, 190, 127-132.

[13] Takasuka, T.E., Book, A.J., Lewin, G.R., Currie, C.R., et al., Aerobic deconstruction of cellulosic biomass by an insect-associated Streptomyces, Sci. Rep. 2013, 3, 1030.

[14] Padilla-Reynaud, R., Simao-Beaunoir, A.-M., Lerat, S., Bernards, M.A., et al., Suberin Regulates the Production of Cellulolytic Enzymes in Streptomyces scabiei, the Causal Agent of Potato Common Scab, Microbes Environ. JSME. 2015, 30, 245-253.

[15] Francis, I.M., Jourdan, S., Fanara S., R. Loria, et al., The Cellobiose Sensor CebR Is the Gatekeeper of Streptomyces scabies Pathogenicity, mBio. 2015, 6, e02018-14. 
[16] Kieser, T., Bibb, M.J., Buttner, M.J., Chater, K.F., et al., Practical Streptomyces Genetics, John Innes Center Norwich, The John Innes Foundation, 2000.

[17] CEN (1996) EN 113 Wood preservatives. Test method for determinating the protective effectivness against wood destroying basidiomycetes. Determination of the toxic values. European Committee for Standardisation, Brussels, Belgium

[18] Sambrook, J., Russell, D.W., Molecular Cloning: A Laboratory Manual, Cold Spring Harbor Laboratory Press, Cold Spring Harbor, New York, 2001.

[19] Ingram, C., Brawner, M., Youngman, P., Westpheling, J., xylE functions as an efficient reporter gene in Streptomyces spp.: use for the study of galP1, a catabolite-controlled promoter, J. Bacteriol. 1989, 171, 6617-6624.

[20] Bradford, M.M., A rapid and sensitive method for the quantitation of microgram quantities of protein utilizing the principle of protein-dye binding, Anal. Biochem. 1976, 72, 248-254.

[21] Bibb, M.J., J. White, J.M. Ward, G.R. Janssen, The mRNA for the 23S rRNA methylase encoded by the ermE gene of Saccharopolyspora erythraea is translated in the absence of a conventional ribosome-binding site, Mol. Microbiol. 1994, 14, 533-545.

[22] Schmitt-John, T., Engels, J.W., Promoter constructions for efficient secretion expression in Streptomyces lividans, Appl. Microbiol. Biotechnol. 1992, 36, 493-498.

[23] Bereza-Malcolm, L.T., Mann, G., Franks, A.E., Environmental sensing of heavy metals through whole cell microbial biosensors: a synthetic biology approach, ACS Synth. Biol. 2015, 4.

[24] Park, M., Tsai, S.-L., Chen, W., Microbial Biosensors: Engineered Microorganisms as the Sensing Machinery, Sensors. 2013, 13, 5777-5795.

[25] Ludwig, R., Harreither, W., Tasca F., Gorton, L., Cellobiose Dehydrogenase: A Versatile Catalyst for Electrochemical Applications, ChemPhysChem. 2010, 11, 2674-2697.

[26] Hanahan, D., Studies on transformation of Escherichia coli with plasmids, J. Mol. Biol. 1983, 166, 557-580.

[27] Simon, R., Priefer, U., Pühler, A., A Broad Host Range Mobilization System for In Vivo Genetic Engineering: Transposon Mutagenesis in Gram Negative Bacteria, Nat. Biotechnol. 1983, 1, 784-791.

[28] Bruton C.J., Guthrie E.P., Chater K.F., Phage Vectors that Allow Monitoring of Transcription of Secondary Metabolism Genes in Streptomyces, Nat. Biotechnol. 1991, 9, 652-656.

[29] Thibessard, A., Haas, D., Gerbaud, C., Aigle, B., et al., Complete genome sequence of Streptomyces ambofaciens ATCC 23877, the spiramycin producer, J. Biotechnol. 214 (2015) 117-118.

[30] Rückert, C., Albersmeier, A., Busche, T., Jaenicke, S., et al., Complete genome sequence of Streptomyces lividans TK24, J. Biotechnol. 2015, 199, 21-22.

[31] Wilkinson, C.J., Hughes-Thomas, Z.A., Martin, C.J., et al., Increasing the efficiency of heterologous promoters in actinomycetes, J. Mol. Microbiol. Biotechnol. 2002, 4, 417-426. 


\section{TABLE}

485 Table 1. Bacterial strains and vector used in this study

\begin{tabular}{llc}
\hline Strain or plasmid & Relevant characteristic(s) & Reference \\
\hline E. coli DH5a & supE44 $\triangle l a c U 169(\Phi 80 l a c Z \Delta M 15)$ hsdR17 recA1 endA1 gyrA96 thi-11 relA1 & {$[26]$} \\
E. coli S17.1 & recA pro hsdR RP4-2-Tc::Mu-Km::Tn7 & {$[27]$} \\
S. coelicolor KC900 & actl::KC900 hisA1 uraA1 strA1 pgl SCP1 SCP2 & {$[28]$} \\
S. ambofaciens ATCC 23877 & Wild-type strain & {$[29]$} \\
S. lividans TK24 & Wild-type strain & {$[30]$} \\
S. coelicolor M145 & SCP1 SCP2 & {$[15]$} \\
Streptomyces sp. S9N29 & Environmental wild-type strain & {$[9]$} \\
Streptomyces sp. S4N27 & Environmental wild-type strain & {$[9]$} \\
Streptomyces sp. S6N6 & Environmental wild-type strain & {$[9]$} \\
Streptomyces sp. S2N2 & Environmental wild-type strain & {$[9]$} \\
Streptomyces sp. S9N14 & Environmental wild-type strain & {$[9]$} \\
Streptomyces sp. S1N3 & Environmental wild-type strain & {$[9]$} \\
Streptomyces sp. S4N22 & Environmental wild-type strain & {$[9]$} \\
plB139 & oriT attP int aac(3)IV PermE* & {$[31]$} \\
\hline
\end{tabular}

486

487

488

489

490

491

492

493

494

495

496

497

498

499 
503 Figure 1: Design and construction flowchart of the biosensor

504 A) Schematic illustration of the cellobiose biosensor system. The biosensor construction consists in a Streptomyces conserved cebR-box sequence cloned between the constitutive promoter PermE* and the reporter gene xylE. Once integrated in a Streptomyces genome harboring an endogenous CebR able to recognize the cebR-box, this transcriptional repressor will bind it and prevents the xylE expression. The presence of cellobiose alleviated the CebR binding on the cebR-box and enables the transcription of $x y / E$. In presence of catechol, the XylE protein will emit a yellow compound revealing the presence of cellobiose. B) Flowchart of the biosensor construction. I. For the construction of the biosensor, the reporter gene xylE was amplified from Streptomyces coelicolor KC900 with the Biosens_F and Biosens_R primers (955 bp). The primer Biosens_F was designed from 5' to 3 ' with a $B a m \mathrm{HI}$ site as a future cloning site in plB139. The black box symbolizes the cebR-box, the light grey box a Ribosome Binding Site (RBS) and the white arrow the beginning of the $x y l E$ gene. The reverse primer Biosens_R has from 5' to 3', an EcoRI site as future cloning site with pIB139 and the white arrow the end of the $x y I E$ gene. II. After amplification, the PCR product was cloned in the plB139 plasmid that enables the integration of the construction into Streptomyces chromosome.

PermE* ${ }^{*}$ constitutive promotor; ori ${ }_{p u c}$ : replicative origin in E. coli; Apra ${ }^{\mathrm{R}}$ : Apramycine gene resistance; oriT: transfert origin, Int: integrase gene; MCS: Multiple cloning site.

Figure 2: Illustration of the detection of the cellobiose by the biosensor The S4N27 biosensor strain was grown for 2 days on $\mathrm{HT}^{*}$ plates without cellobiose in $\mathrm{A}$ ) or with cellobiose at $5 \mathrm{~g} \cdot \mathrm{L}^{-1}$ in $\left.\mathrm{B}\right)$. After catechol addition the yellow color is only observed in presence of cellobiose.

Figure 3: Detection threshold of cellobiose by the biosensor.

The experiments were performed in $\mathrm{HT}^{*}$ medium complemented with increasing cellobiose concentrations ranging from $1.5 \mu \mathrm{M}$ to $15000 \mu \mathrm{M}$. For each concentration, the results are expressed as the ratio of the $x y l E$ activity measured in presence and in absence of cellobiose

532 (background signal). An activity ratio of 1 (represented by the black horizontal line) corresponds to an activity equivalent to the background signal. Results for each concentration corresponded to three independent cultures. Error bars represent the standard 
535 deviation. Significant differences of signal intensity between the different conditions and the 536 background signal are represented by asterisks and were determined with the Student's $t$ 537 test $(P<0.05)$.

538

539

Figure 4: Detection of the wood degradation by a lignolytic fungus.

Wood leachates were obtained from a spruce wood stick infected for 7 weeks by Poria placenta (black box) and from a non-infected control (white box). The results correspond to five independent biosensor assays and are expressed as the relative expression with the non-infected condition. Thus, the activity ratio of the non-infected spruce wood condition has a value of one and corresponds to the background signal. The significant difference between the two experimental conditions was attested by a Student's $t$-test $(P<0.05)$ and is symbolized by an asterisk.

\section{Acknowledgement}

This work was funded by the Région Lorraine and the French National Research Agency through the Laboratory of Excellence ARBRE (ANR-11- LABX-000-01).

\section{Conflict of interest.}

The authors declare no financial or commercial conflict of interest. 
Figure 1

A)

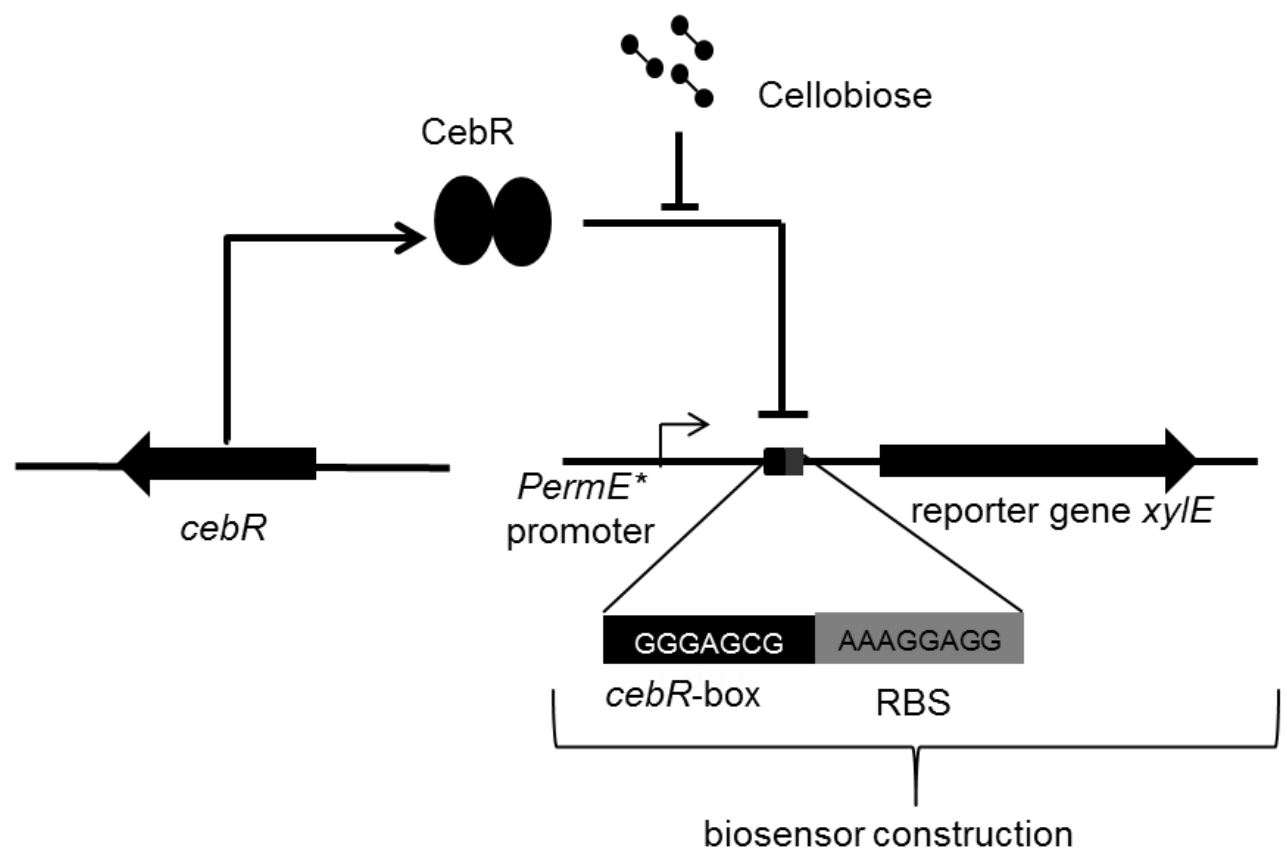

B)

I.

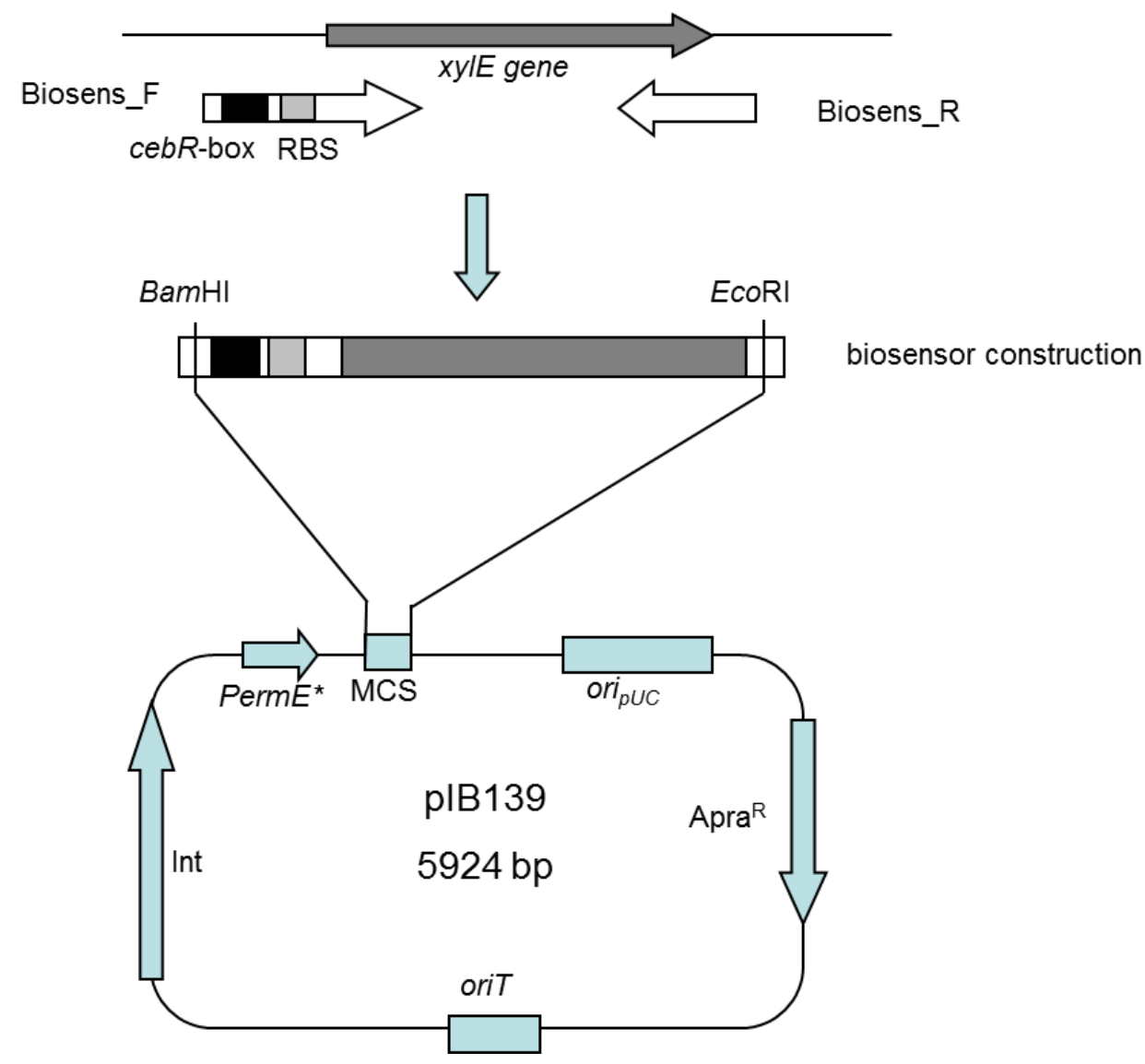


610 Figure 2

A

\section{B}
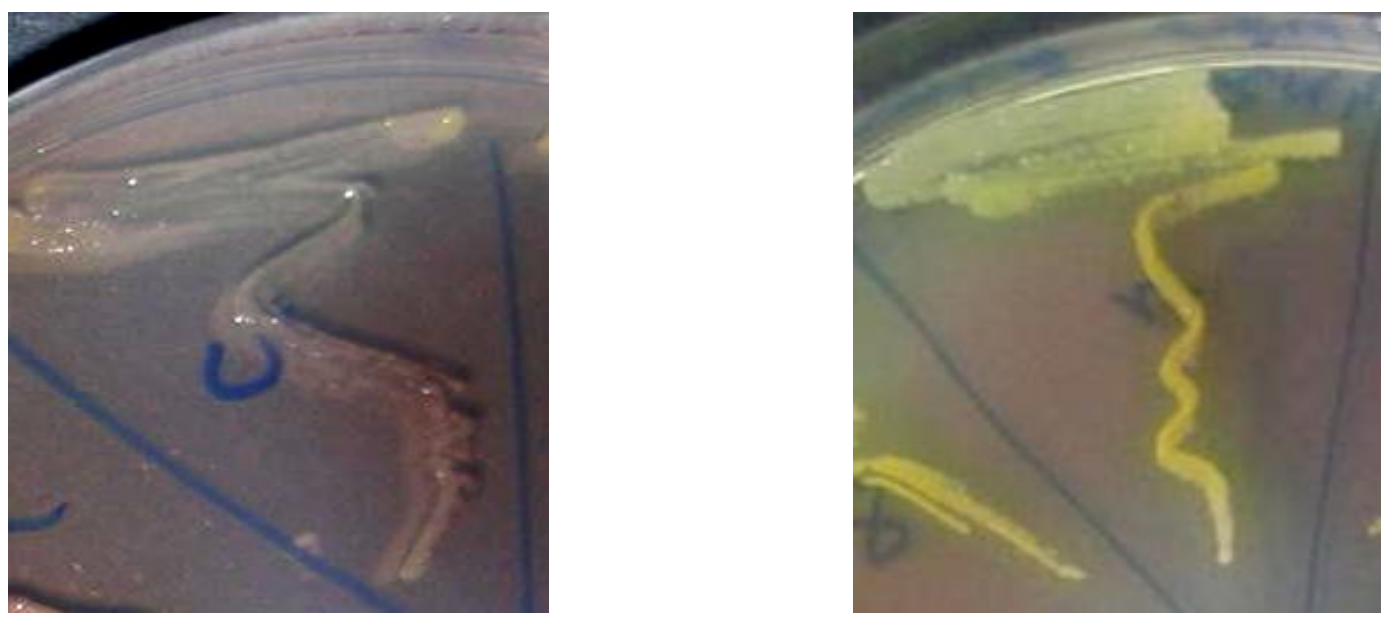

- cellobiose

+ cellobiose 
611 Figure 3

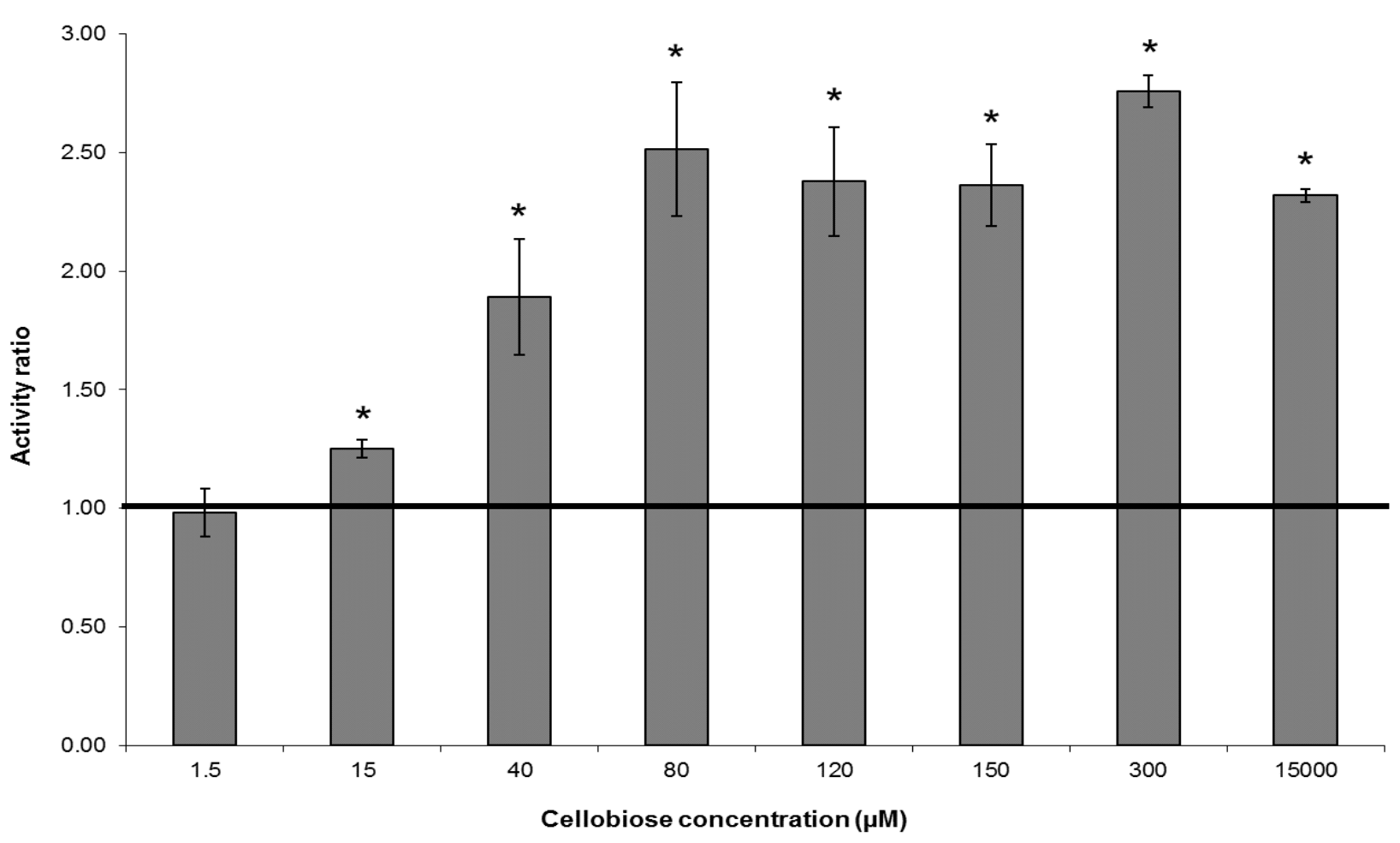


$612 \quad$ Figure 4

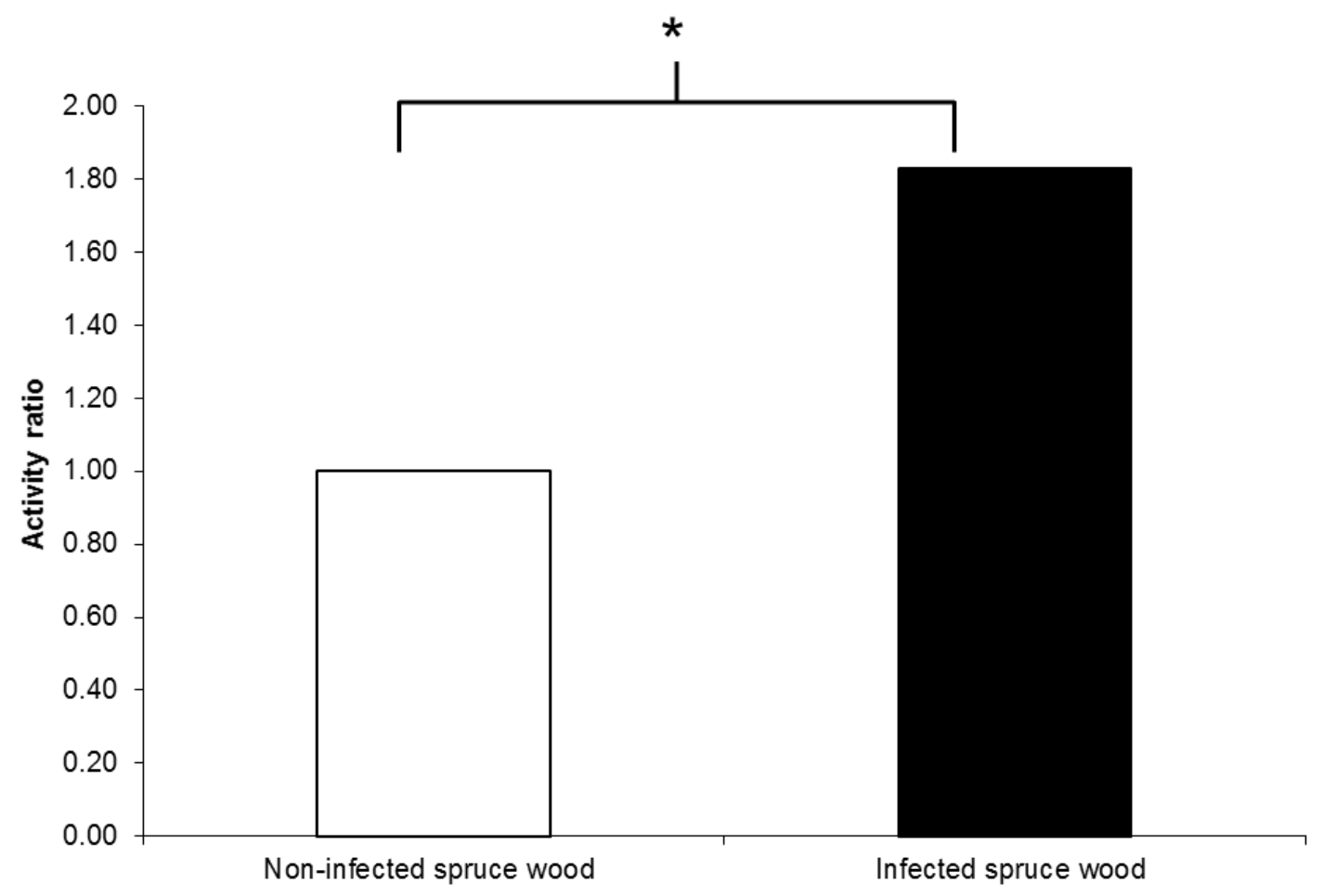

613 